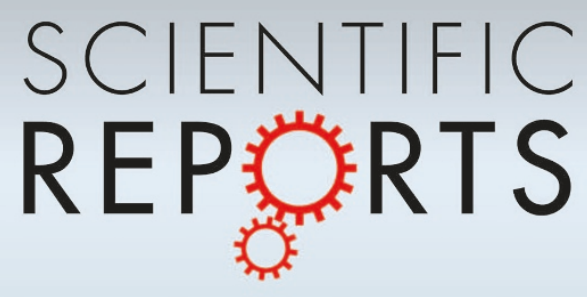

\title{
OPEN A unique platinum-graphene hybrid structure for high activity and durability in oxygen reduction reaction
}

SUBJECT AREAS:

ELECTROCATALYSIS

MATERIALS CHEMISTRY

SYNTHESIS AND PROCESSING

GRAPHENE

\author{
Chengming Wang ${ }^{1 *}$, Liang Ma ${ }^{2 *}$, Lingwen Liao² ${ }^{2}$ Song Bai ${ }^{2}$, Ran Long ${ }^{2}$, Ming Zuo' \& Yujie Xiong ${ }^{1,2}$
}

Received

4 April 2013

'Hefei National Laboratory for Physical Sciences at the Microscale, University of Science and Technology of China, Hefei, Anhui 230026, P. R. China, ${ }^{2}$ Collaborative Innovation Center of Chemistry for Energy Materials, School of Chemistry and Materials Science, University of Science and Technology of China, Hefei, Anhui 230026, P. R. China.

Accepted

19 August 2013

Published

3 September 2013

It remains a grand challenge to achieve both high activity and durability in Pt electrocatalysts for oxygen reduction reaction (ORR) in fuel cells. Here we develop a class of Pt highly concave cubic (HCC) nanocrystals, which are enriched with high-index facets, to enable high ORR activity. The durability of HCC nanocrystals can be significantly improved via assembly with graphene. Meanwhile, the unique hybrid structure displays further enhanced specific activity, which is 7 -fold greater than the state-of-the-art $\mathrm{Pt} / \mathrm{C}$ catalysts. Strikingly, it exhibits impressive performance in terms of half-wave potential $\left(E_{1 / 2}\right)$. The $E_{1 / 2}$ of $0.967 \mathrm{~V}$ at the $\mathrm{Pt}$ loading as low as $46 \mu \mathrm{g} \mathrm{cm}^{-2}$, which stands as $63 \mathrm{mV}$ higher than that of the Pt/C catalysts,

Correspondence and requests for materials should be addressed to Y.J.X. lyjixiong@ustc. edu.cn)

* These authors contributed equally to this work. is slightly superior to the record observed for the most active porous $\mathrm{Pt}$-Ni catalyst in literature. This work paves the way to designing high-performance electrocatalysts by modulating their surface and interface with loading substrates.

W ith the increasing needs for clean energy, electrochemical reactions have become indispensable components in various energy-conversion systems. Among the reactions, cathodic oxygen reduction reaction (ORR), $\mathrm{O}_{2}+4 \mathrm{H}^{+}+4 \mathrm{e}^{-} \rightarrow 2 \mathrm{H}_{2} \mathrm{O}$, represents a highly important process in proton exchange membrane fuel cells (PEMFC) ${ }^{1-4}$. The slow kinetics and high over-potential (300 mV in most cases), which are two typical characteristics of the ORR reaction, make it necessary to employ catalysts to improve the ORR activity in a low-temperature PEMFC. Among various parameters, the half-wave potential $\left(\mathrm{E}_{1 / 2}\right)$ of polarization currents is an important one to assess the performance of ORR catalysts. The value of $E_{1 / 2}$ indicates the capability of catalysts in reducing the ORR over-potential. In fact, the design of novel electrocatalysts has become a central research theme in efforts to increase their $\mathrm{E}_{1 / 2}$ value. Thus far, a great deal of effort has been directed toward modifying the structures of conventional platinum $(\mathrm{Pt}$ ) catalysts, which includes alloying process to formulate $\mathrm{Pt}-$ $\mathrm{M}$ nanocatalysts ${ }^{5-12}$, coating of Pt monolayer on non-Pt nanoparticles to form M@Pt core-shell catalysts ${ }^{13-17}$, synthesis of porous Pt-M catalysts ${ }^{18}$, and development of non-Pt catalysts ${ }^{19-22}$. This series of investigations suggests that specific Pt-M alloy catalysts may possess high activities than pure Pt catalysts in the ORR, as the alloying process enables more versatility in optimizing the structures of catalysts for enhanced electrocatalytic performance ${ }^{12}$. Notably, it has been a milestone that porous Pt-Ni alloys exhibit an $\mathrm{E}_{1 / 2}$ of $0.96 \mathrm{~V}$ in the ORR ${ }^{18}$. The incorporation of $\mathrm{Ni}$ into Pt catalysts has been demonstrated to maneuver their electronic structure and arrangement of surface atoms, and in turn, enhance their ORR activities ${ }^{12,23}$.

Despite the progress in various alloys, it is well established that surface structure is a critical parameter to tune the activities of electrocatalysts in the $\mathrm{ORR}^{24,25}$. Owing to this fact, it represents a major trend of research to establish the relationship between Pt surface structure and ORR activity. In practice, it would be much more sophisticated to elucidate the relationship in the case of alloy electrocatalysts, as many factors may interplay in this complex system. From the perspective of fundamental understanding, it is imperative to perform the investigation by employing pure Pt nanocrystals as a simpler model system. Ideally, this investigation is expected to enable the activities of pure Pt catalysts to reach the level comparable to their alloy counterparts with the best performance, under the circumstance that their surface structures can be well designed. As long as the structure-activity relationship is established, it can be further implemented in the alloy systems, and in turn, will definitely bring the performance of alloy catalysts to another higher level.

Previous research has provided some insights into the electrocatalyst design based on surface control. The investigations, which have been mostly focused on the Pt single-crystal electrodes in a perchloric acid system, 
indicate that the $\mathrm{E}_{1 / 2}$ of ORR polarization on $\mathrm{Pt}$ low-index facets is in the order of $(110)>(111)>(100)^{23,24}$. It is worth noting that the ORR activities can be greatly enhanced on the interlaced (111)-(100) Pt surfaces at the nanoscale, according to the study using the $\mathrm{Pt}$ model electrodes ${ }^{25}$. Most likely a synergetic effect between various facets promotes the overall performance of Pt catalysts: (100) facets can facilitate the adsorption of molecular oxygen $\left(\mathrm{O}_{2}\right)$, while the adsorbed $\mathrm{O}_{2}$ may diffuse onto the neighbored (111) facets for successive reactions on which $\mathrm{OH}$ species have weaker adsorption ${ }^{25}$. As a result, it demonstrates that stepped facets such as (211), (331) and (311) have higher ORR activities than the low-index facets ${ }^{23,24}$. Thus formulating high-index facets represents a powerful tool to enhance the activities of catalysts made of pure $\mathrm{Pt}$, owing to the unique function of stepped surface. In the past years, considerable efforts have been made to synthesize Pt nanocrystals with well-defined surface facets $^{26-29}$; however, their ORR activities are still not comparable to the milestone work in alloy catalysts.

To further improve the performance of pure Pt catalysts, it is needed to develop a new synthetic system for Pt nanocrystals enclosed by high-index facets and understand the relationship between ORR activities and surface stepped structures. Herein, we report a unique Pt nanostructure with a highly concave cubic (HCC) profile that is enriched with $\{311\}$ high-index facets. The nanocrystals are 4.8-fold and 6.0-fold more active for the ORR than the state-of-theart $\mathrm{Pt} / \mathrm{C}$ catalysts in terms of mass-specific and area-specific activities, respectively. In the assessment of $E_{1 / 2}$ value, our nanocrystals exhibit $0.960 \mathrm{~V}$ at a Pt loading weight as low as $46 \mu \mathrm{g} \mathrm{cm}^{-2}$, which is very comparable to that observed for the most active porous $\mathrm{Pt}-\mathrm{Ni}$ catalyst in literature ${ }^{18}$. In comparison with the Pt-Ni catalyst, the Pt loading amount in our work is relatively lower. This work clearly demonstrates that high ORR activities and low material costs can be both achieved by formulating unique surface structures without the need of forming alloys. Further investigation shows that the durability of electrocatalysts (up to 10000 cycles) can be further improved by constructing hybrid structures between Pt nanocrystals and graphene, while their high activities are further slightly enhanced. Graphene is a two-dimensional single-layer carbon material with high in-plane conductivity and mechanical strength ${ }^{30-35}$, which has been demonstrated as a good substrate candidate for improving catalyst durability ${ }^{36,37}$. This work provides another strategy for designing high-performance and low-cost catalysts for the ORR reaction, in addition to the popularly investigated alloying technique.
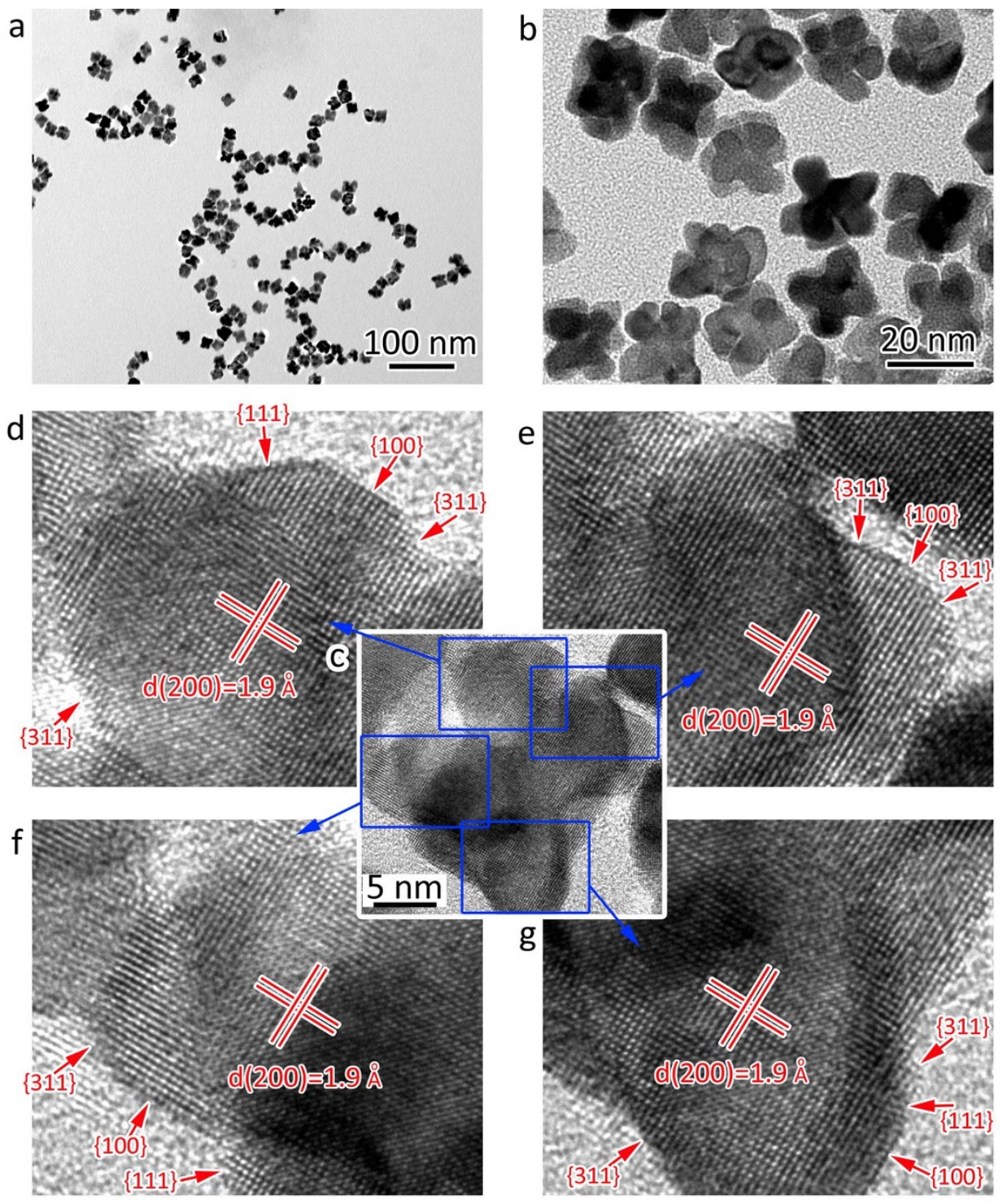

Figure 1 TEM characterizations of Pt HCC nanocrystals. (a), (b), TEM images at different magnifications. (c-g), HRTEM images of a HCC nanocrystal, focused on each single corner. 


\section{Results}

The Pt HCC nanocrystals (Pt HCCs) were synthesized by modifying the protocol that we have previously developed for the synthesis of branched nanocrystals ${ }^{38}$. In the previous protocol, the branched growth of Pt nanocrystals was mainly maneuvered by oxidative etching. Here, the strength of oxidative etching is reduced by protecting the reaction system with a mixed gas of Ar and air at the ratio of $0.8-$ 1.2. As a result, the product of Pt nanocrystals can be tuned to have a better-defined shape. Transmission electron microscopy (TEM) images in Figures 1a and 1b reveal that more than 95\% of the nanocrystals have a highly concave cubic profile. In order to identify the structure of nanocrystals, we have employed high-resolution TEM (HRTEM) to characterize the sample. Figures $1 \mathrm{c}-\mathrm{g}$ show the images taken from each corner of a HCC nanocrystal, indicating: $i$ ) the center of the nanocrystal is a $\{100\}$-oriented cube; $i$ ) the exposed facets are composed of $\{111\},\{100\}$ and $\{311\}$.

The high-index $\{311\}$ facets on surface may make the nanocrystals particularly active in electrocatalysis. To investigate their activity in the ORR reaction, the sample was coated on a glassy carbon (GC) rotating disk electrode (RDE) after specific cleaning procedures. Xray photoelectron spectroscopy (XPS) has confirmed that the sample is free of contaminants prior to the electrochemical measurements (see Figure S1). Figure 2a shows cyclic voltammetry (CV) curves of Pt HCC nanocrystals recorded at room temperature in $\mathrm{N}_{2}$-purged $0.1 \mathrm{M} \mathrm{HClO}_{4}$ solution at a scan rate of $20 \mathrm{mV} \mathrm{s}^{-1}$, benchmarked against the $10-\mathrm{nm}$ commercial Pt/C catalyst (E-TEK, Pt: $40 \%$ wt., $10 \mathrm{~nm}$ ). The $\mathrm{CV}$ curves normalized by electrochemically active areas (ECAs) exhibit two potential regions: the one between 0.05 and $0.4 \mathrm{~V}$

a

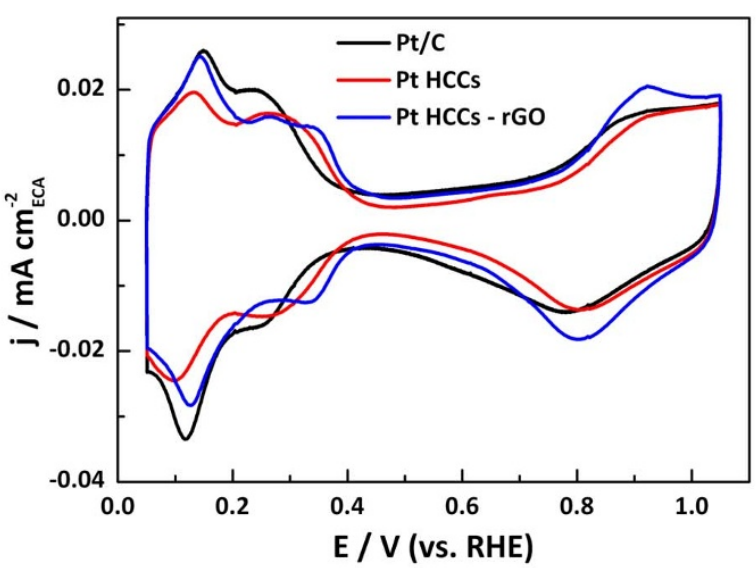

C

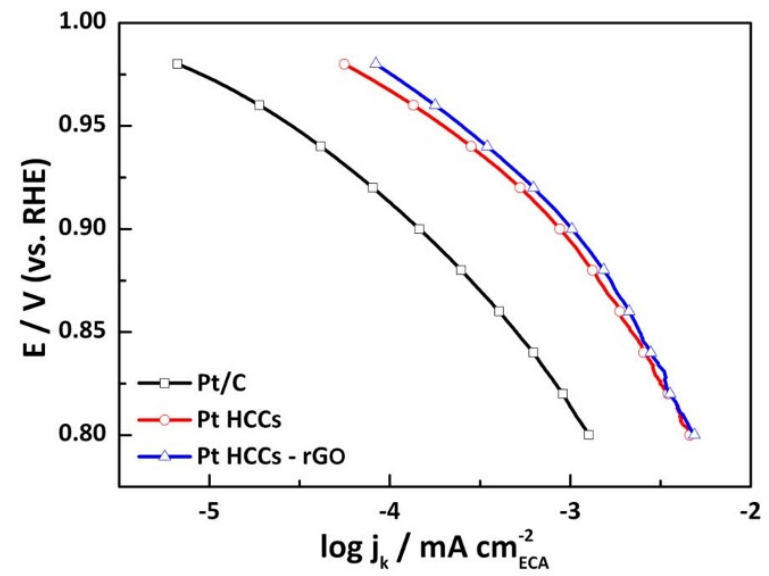

that can be attributed to the adsorption/desorption processes of underpotentially deposited hydrogen, and the other beyond $0.6 \mathrm{~V}$ that corresponds to the formation of a layer of adsorbed hydroxyl species $^{4,12}$. As a result, the ECA can be calculated by accumulating the charge in the $0.05-0.4 \mathrm{~V}$ region assuming a value of $220 \mu \mathrm{C} \mathrm{cm}^{-2}$ for the adsorption of a hydrogen monolayer ${ }^{39,40}$. The ECA values of the three catalyst samples, which are calculated from raw blank CVs (Figure S2), are listed in Table S1. The ECA value of the commercial $10-\mathrm{nm} \mathrm{Pt} / \mathrm{C}$ catalysts $\left(9.12109 \mathrm{~cm}^{2}\right)$ is higher than that of our $\mathrm{Pt}$ HCCs catalysts $\left(7.30408 \mathrm{~cm}^{2}\right)$. Given that the size of Pt HCCs is $\sim 18 \mathrm{~nm}$, this result is well consistent with the general understanding that the ECA of nanocatalysts becomes larger as the particle size decreases ${ }^{41-43}$.

In order to evaluate the electrocatalytic performance of Pt catalysts, ORR measurements were further performed in $\mathrm{O}_{2}$-saturated $0.1 \mathrm{M} \mathrm{HClO}_{4}$ solution using a GC RDE with Pt loading at $46 \mu \mathrm{g} \mathrm{cm}^{-2}$ at room temperature. Figure $2 \mathrm{~b}$ shows the ORR polarization curves for the Pt HCC nanocrystals and commercial Pt/C catalysts. The half-wave potential $\left(\mathrm{E}_{1 / 2}\right)$ of Pt HCC nanocrystals has been determined to be $0.960 \mathrm{~V}$, which is $56 \mathrm{mV}$ higher than that of $\mathrm{Pt} / \mathrm{C}$ catalysts (see Table 1). This value is very comparable to that observed for the most active porous $\mathrm{Pt}-\mathrm{Ni}$ catalyst in literature ${ }^{18}$; however, the $\mathrm{Pt}$ loading in our case is relatively lower.

The kinetic current can be calculated based on the KouteckyLevich equation:

$$
\frac{1}{i}=\frac{1}{i_{k}}+\frac{1}{i_{d}}
$$

b
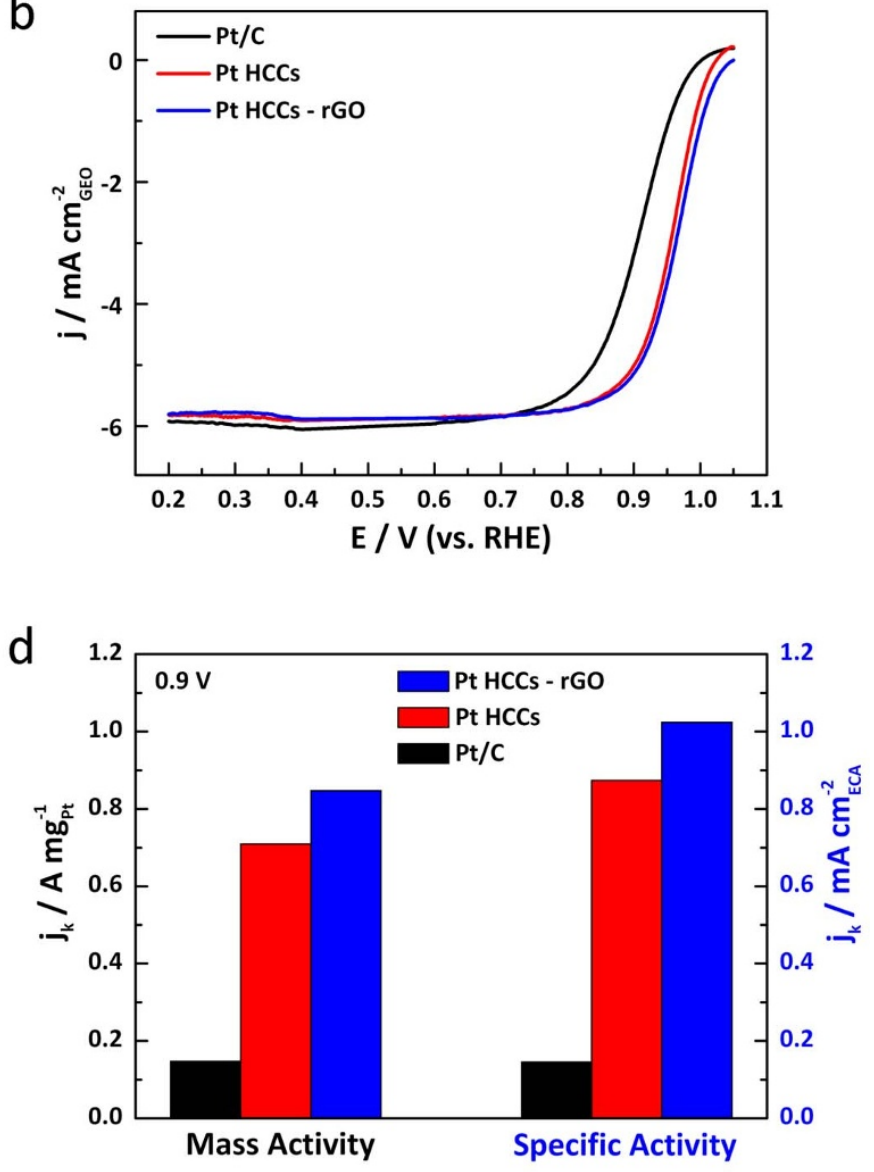

Figure 2 Comparison of electrochemical activities of Pt HCCs and Pt HCCs-rGO samples with Pt/C. (a), CV curves recorded in $\mathrm{N}_{2}$-saturated 0.1 M $\mathrm{HClO}_{4}$ solution at a scan rate of $20 \mathrm{mV} \mathrm{s}^{-1}$. (b), ORR polarization curves in $\mathrm{O}_{2}$-sataurated $0.1 \mathrm{M} \mathrm{HClO}_{4}$ solution at room temperature. The potential scan rate was $20 \mathrm{mV} \mathrm{s}^{-1}$, and the electrode rotation speed was $1600 \mathrm{rpm}$. (c), Tafel plots. (d), Mass activity and specific activity at $0.9 \mathrm{~V}$ versus RHE. 
Table 1 | Half-wave potentials $\left(E_{1 / 2}\right)$ of the ORR on GC RDEs that were prepared with the three catalysts

\begin{tabular}{lccc} 
Catalysts & Pt/C & Pt HCCs & Pt HCCs-rGO \\
\hline $\mathrm{E}_{1 / 2}$ Initial $(\mathrm{V}$ vs. RHE) & 0.904 & 0.960 & 0.967 \\
$\mathrm{E}_{1 / 2 \text { After } 10000 \text { cycles }}$ & 0.893 & 0.943 & 0.959 \\
$\quad(\mathrm{~V}$ vs. RHE) & & & \\
\hline
\end{tabular}

where $i$ is the experimentally measured current, $i_{d}$ is the diffusionlimiting current, and $i_{k}$ is the kinetic current. The mass activity and specific activity can be obtained by normalizing the kinetic currents with Pt loading and ECA, respectively. As a result, the Tafel plots have been acquired based on the relationship between the specific activity and the potentials. Figure $2 \mathrm{c}$ shows the Tafel plots of Pt HCC nanocrystals and $\mathrm{Pt} / \mathrm{C}$ catalysts revealing their specific activities. The plots of both catalysts exhibit characteristics that have slopes of about $-120 \mathrm{mV} \mathrm{dec}{ }^{-1}$ between $0.83-0.90 \mathrm{~V}$ and $-60 \mathrm{mV} \mathrm{dec}{ }^{-1}$ between $0.90-0.98 \mathrm{~V}$, respectively. At potentials below $0.98 \mathrm{~V}$ versus RHE, the slopes of the curves at a fixed potential are approximately equal, indicating the same reaction mechanism over the majority of the potential range. In addition, the Tafel plots reveal that the Pt HCC nanocrystals have higher kinetic currents than the $\mathrm{Pt} / \mathrm{C}$ at a given potential, implying the higher activity of Pt $\mathrm{HCCs}^{18,44,45}$. The ORR mass activity and specific activity of different catalysts at $0.9 \mathrm{~V}$ are summarized in Figure 2d. It shows that the mass and specific activities of Pt HCC nanocrystals reach $-0.709 \mathrm{~A} \mathrm{mg}_{\mathrm{Pt}^{-1}}$ and $-0.874 \mathrm{~mA} \mathrm{~cm}^{-2}$, which are 4.8 times and 6.0 times greater than

a

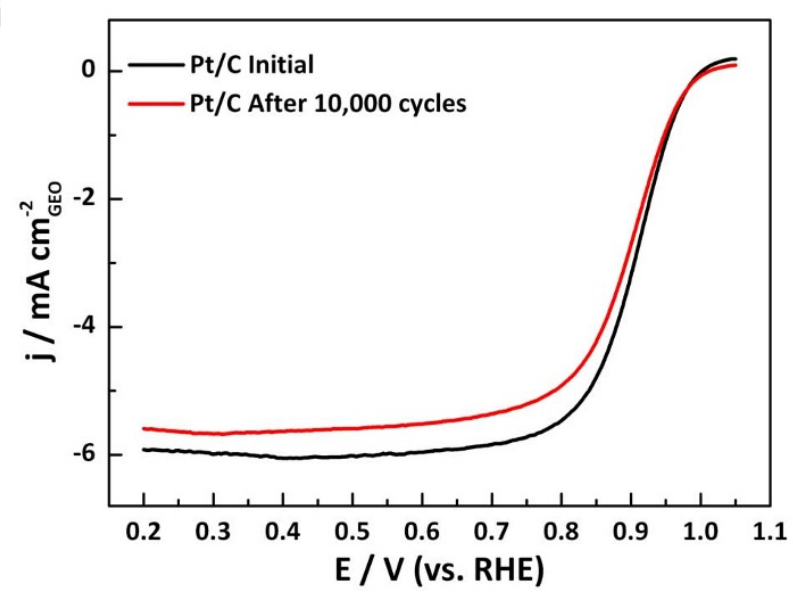

C

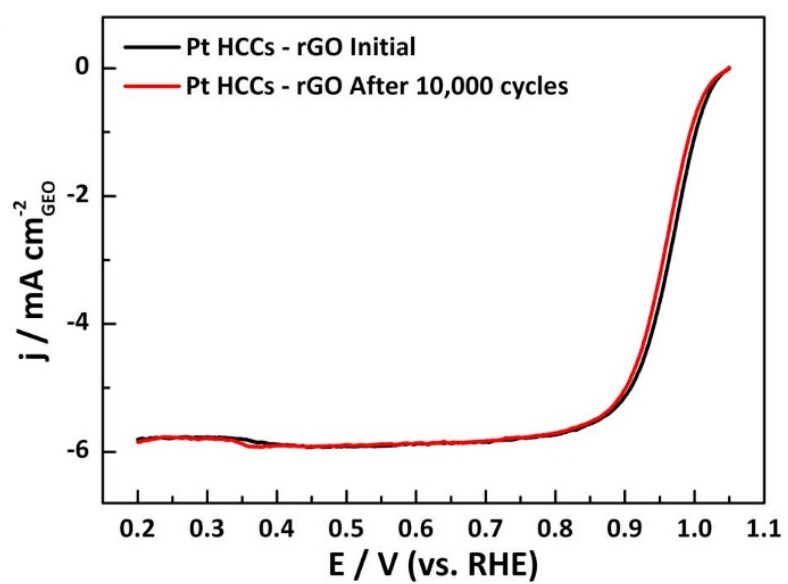

those of Pt/C catalysts, respectively. The superior activity of Pt HCCs sample is better observed in the entire region between 0.83 and $1.05 \mathrm{~V}$ (see Figure S3).

More detailed investigations on the ORR properties have been performed by altering the rotation speed of RDE. Figure S4 shows the ORR polarization curves at different rotation speeds that were normalized by the geometrical area of electrodes. The curves display the diffusion-limiting current region from 0.4 to $0.8 \mathrm{~V}$ and the mixed kinetic-diffusion control region between $\sim 0.8$ and $\sim 1.0 \mathrm{~V}$. According to the Equation (2) below, it is feasible to deduce the Equation (3) by applying the kinematic viscosity measured by the Hsueh group (i.e., $v=0.0087 \mathrm{~cm}^{2} \mathrm{~s}^{-1}$ ) and the value of $C o^{*} D o^{2 / 3}=$ $7.6 \times 10^{-10} \mathrm{~mol} \mathrm{~cm}^{-5 / 3} \mathrm{~s}^{-2 / 346}$

$$
\begin{aligned}
& i_{d}=-0.62 n F A D_{O}^{2 / 3} \omega^{1 / 2} v^{-1 / 6} C_{O}^{*} \\
& i_{d}=-0.41 \omega^{1 / 2} \mathrm{~mA} \cdot \mathrm{cm}^{-2} \cdot \mathrm{s}^{-1 / 2}
\end{aligned}
$$

Here, $n$ is the number of transferred electrons in the reaction, $F$ is the Faraday constant, $A$ is the geometrical area, $D o$ is the diffusion coefficient, $\omega$ is the rotation speed, $v$ is the kinematic viscosity of solution, and $\mathrm{Co}$ is the bulk concentration of the reagents in the solution. As a result, we can make K-L plots for the samples at $0.5 \mathrm{~V}$ (see Figure S5). The slopes of plots for Pt HCC nanocrystals and $\mathrm{Pt} / \mathrm{C}$ catalysts are -0.450 and $-0.466 \mathrm{~mA} \mathrm{~cm}^{-2} \mathrm{~s}^{-1 / 2}$, respectively, which agrees well with the theoretical value for the four-electron transfer process $\left(-0.410 \mathrm{~mA} \mathrm{~cm}{ }^{-2} \mathrm{~s}^{-1 / 2}\right)$, indicating that the ORR reaction occurring on both the catalysts is four-electron transfer ${ }^{46}$.

b

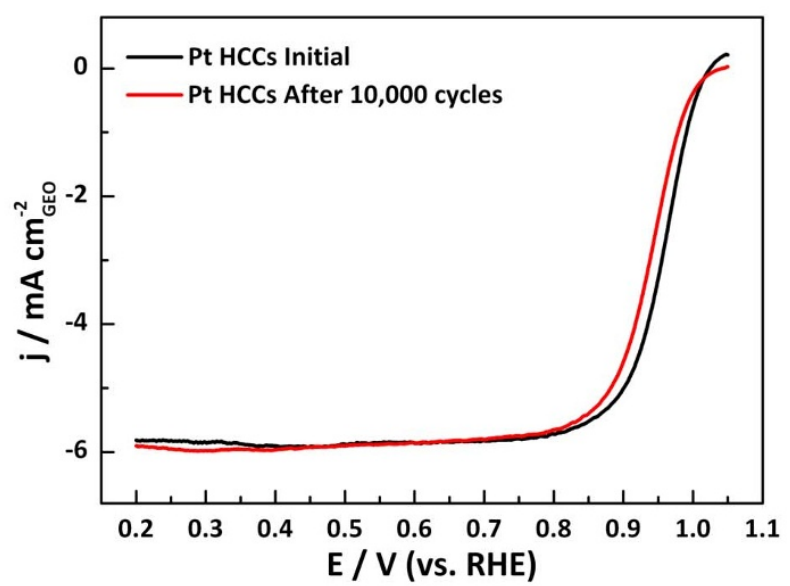

d

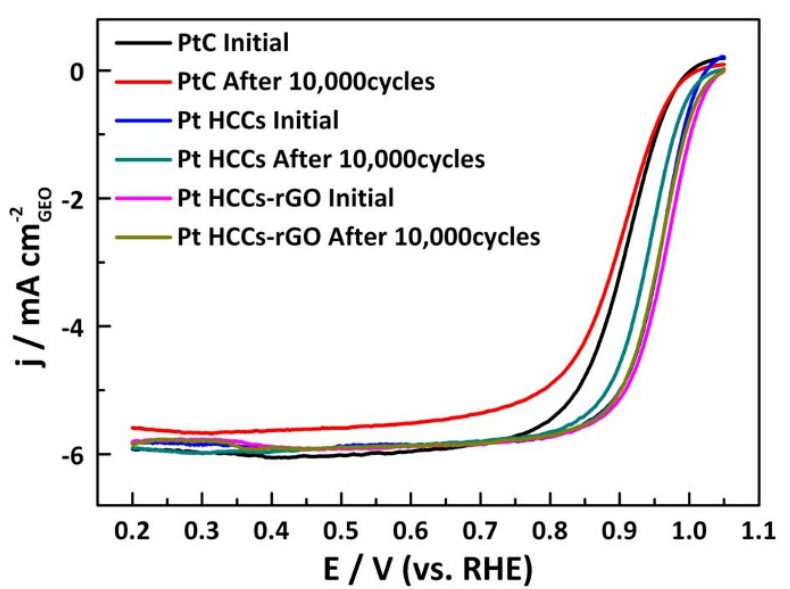

Figure $3 \mid$ Durability tests of samples by running the ORR reaction for 10000 cycles. (a), commercial Pt/C catalysts. (b), Pt HCCs. (c) Pt HCCs-rGO. (d) Comparison of all the three samples. 
In addition to the activity of catalysts, durability is another important parameter to evaluate their performance in the ORR. In order to assess their ability to sustain activity, we have performed durability tests by applying cyclic potential sweeps between 0.60 and $1.05 \mathrm{~V}$ (which causes surface oxidation/reduction cycles of $\mathrm{Pt}$ ) at a sweep rate of $50 \mathrm{mV} \mathrm{s}^{-1}$ and a rotation speed of $1600 \mathrm{rpm}$ in $\mathrm{O}_{2}$-saturated $0.1 \mathrm{M} \mathrm{HClO}_{4}$ solution at room temperature. After 10000 cycles, the $\mathrm{Pt} / \mathrm{C}$ catalysts show significantly reduced diffusion-limiting currents (see Figure 3a). It indicates that part of catalysts have fallen off from the $\mathrm{RDE}$, raising the concern that the $\mathrm{Pt} / \mathrm{C}$ has limited binding to the electrode. In contrast, Figure $3 \mathrm{~b}$ shows that the half-wave potential of Pt HCC nanocrystals remains high after the durability tests, with only a negative shift of $17 \mathrm{mV}$ in the mixed kinetic-diffusion control region. This feature should be ascribed to the relatively strong interactions of catalysts with the electrodes. On the other hand, we notice from the TEM studies that the nanocrystals have slightly formulated aggregates although their morphologies have been largely maintained (see Figure S6a). This series of findings highlights the key factors to improve the durability of ORR catalysts: $i$ ) strong binding to the electrodes; $i$ ) high resistance to the morphological changes and aggregations. Given that Pt HCC nanocrystals still exhibit a $17 \mathrm{mV}$ decrease in half-wave potential, it is imperative to develop an approach to further improving their durability by implementing an appropriate substrate.

Graphene has been considered as a promising candidate as substrates for Pt catalysts, in terms of improving their performance regarding the key factors mentioned above. Specifically, graphene can have several merits accordingly: $i$ ) having strong interactions with a broad range of materials including carbon, and possessing capability of suppressing aggregation of nanoparticles ${ }^{47-49}$; ii) improving resistance of metals to electrochemical degradation by at least one order of magnitude when formulating hybrid structures ${ }^{50}$; iii) high Fermi velocity $\left(\mathrm{v}_{\mathrm{F}}=10^{6} \mathrm{~m} \mathrm{~s}^{-1}\right)$ and high in-plane conductivity to enable efficient electron transfer between the catalysts and the electrodes ${ }^{30-35}$. Given the merits of graphene substrates, we have developed a method for assembling Pt HCC nanocrystals on reduced graphene oxide (rGO) where graphene oxide (GO) is functionalized with PVP and further reduced by hydrazine hydrate. Figure 4 shows the TEM characterizations of the as-prepared hybrid structures. As seen from Figures $4 \mathrm{a}$ and $4 \mathrm{~b}$, the Pt nanocrystals are well supported on the rGO substrate, and their highly concave cubic profile is well maintained. HRTEM images in Figure 4c-g reveal that the supported Pt nanocrystals are also $\{100\}$ oriented and enclosed by some $\{311\}$ high-index facets. The nature of rGO is further examined
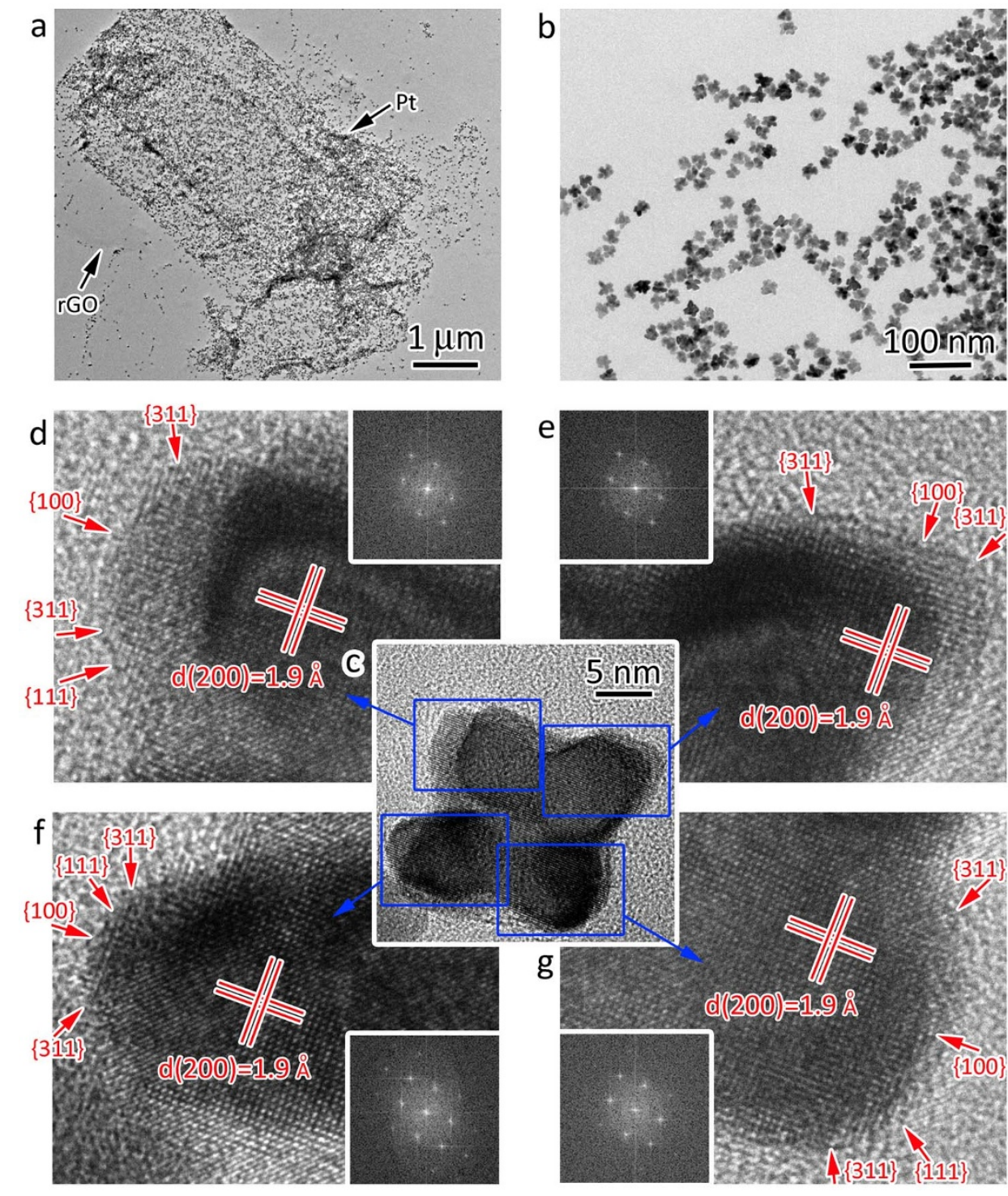

Figure $4 \mid$ TEM characterizations of Pt HCCs-rGO hybrid structures. (a), (b), TEM images at different magnifications. (c-g), HRTEM images of a HCC nanocrystal supported on the rGO substrate, focused on each single corner. The insets show the corresponding Fourier-transformed diffraction patterns. 
by Raman spectroscopy and X-ray photoelectron spectroscopy (XPS). The peaks at 1348 and $1585 \mathrm{~cm}^{-1}$ in the Raman spectra (Figure S7) should be ascribed to the D and G bands of graphene, respectively ${ }^{51,52}$. The $\mathrm{G}$ band represents the graphitic hexagon-pinch mode, while the $\mathrm{D}$ band is related to defects in the curved graphene sheet. The $\mathrm{D} / \mathrm{G}$ intensity ratio increases during the transformation from GO precursor to Pt HCCs-rGO hybrid structure, suggesting a decrease in the average size of the $\mathrm{sp}^{2}$ domains upon the reduction of $\mathrm{GO}^{53}$. In the $\mathrm{C} 1 \mathrm{~s}$ deconvolution XPS spectrum of GO precursor (Figure S8a), four peaks centered at 284.5, 285.5, 286.7 and $288.0 \mathrm{eV}$ are observed, which can be assigned to $\mathrm{C}-\mathrm{C}, \mathrm{C}-\mathrm{OH}$, $\mathrm{C}=\mathrm{O}$, and $\mathrm{O}=\mathrm{C}-\mathrm{O}$ groups, respectively ${ }^{54}$. In contrast, the intensities of all $\mathrm{C} 1$ s peaks that correspond to the $\mathrm{C}$ binding to $\mathrm{O}$ significantly decrease in the spectrum of Pt HCCs-rGO hybrid structure (Figure $\mathrm{S} 8 \mathrm{~b}$ ), suggesting that most of $\mathrm{O}$-containing functional groups have been removed from graphene during the reduction process.

The electrochemical properties of Pt HCCs-rGO hybrid structure have been examined as shown in Figures 2 and S3. At the point that the current density of the polarization curve is zero, the onset-potential for ORR is clearly positively shifted in the order of $\mathrm{Pt} / \mathrm{C}<\mathrm{Pt}$ HCCs $<$ Pt HCCs-rGO, indicating that our Pt HCCs and Pt HCCsrGO catalysts (especially Pt HCCs-rGO) have stronger resistance to oxidation than the commercial $\mathrm{Pt} / \mathrm{C}$ catalyst ${ }^{4,12,55}$. The half-wave potential $\left(\mathrm{E}_{1 / 2}\right)$ of the hybrid structure has been determined to be $0.967 \mathrm{~V}$, which is $7 \mathrm{mV}$ further enhanced as compared with the $\mathrm{Pt}$ HCC nanocrystals (see Table 1). The slightly enhanced activity is also indicated by the Tafel plot of Pt HCCs-rGO sample (see Figure 2c), which generally displays higher kinetic currents than the Pt HCCs at a given potential. Specifically, the Pt HCCs-rGO sample exhibits mass activity of $-0.847 \mathrm{~A} \mathrm{mg}_{\mathrm{Pt}}{ }^{-1}$ and specific activity of $-1.02 \mathrm{~mA}$ $\mathrm{cm}^{-2}$, which are 1.2 times and 1.2 times greater than those of $\mathrm{Pt}$ HCCs, respectively. All the results exclusively demonstrate that the assembly of Pt HCC nanocrystals with rGO enables higher electrocatalytic activity, most likely owing to improved binding to electrodes and superior conductivity. In addition, Figures S4 and S5 suggest that the ORR on the hybrid structure also follows a four-electron transfer mechanism, according to the slope of its $\mathrm{K}-\mathrm{L}$ plot at $-0.445 \mathrm{~mA} \mathrm{~cm}^{-2} \mathrm{~s}^{-1 / 246}$.

Remarkably, the Pt HCCs-rGO hybrid structure exhibits superior durability. The hybrid structure maintains the half-wave potential almost unchanged after 10000 cycles (see Figure 3c), showing improved resistance to performance decay. According to the report by Adizic et al. ${ }^{56}$, the commercial Pt/C catalysts exhibit $-39 \mathrm{mV}$ shift for their half-wave potential after durability tests. It implies that the binding of Pt HCC nanocrystals to graphene is much stronger than that directly to the carbon in $\mathrm{Pt} / \mathrm{C}$. Given the better adhesion to the GC RDE, the use of graphene should lead to the improvement of durability in ORR. Figure S6b reveals that there is no significant change on the structure of Pt HCCs-rGO, and the aggregation between Pt HCC nanocrystals is largely prevented owing to the tension of rGO. On the other hand, as shown in Figure S2, the CV measurements show very minimal ECA loss $(6.8 \%)$ in contrast to the Pt/C (19.5\%) and Pt HCCs (11.2\%). It indicates that most of the Pt HCC nanocrystals are remained on the electrode without distinct aggregations after long-term use, which should be responsible for the durability improvement. The durability comparison of all the three samples is better resolved in Figure S9. After 10000 cycles, the mass activity and specific activity of Pt HCCs-rGO are still as high as $-0.720 \mathrm{~A} \mathrm{mg}_{\mathrm{Pt}}^{-1}$ and $-0.934 \mathrm{~mA} \mathrm{~cm}{ }^{-2}$, respectively, which are 6.3-fold and 6.7-fold higher than those of commercial Pt/C catalysts, suggesting very high activity and durability for ORR. Overall, the results clearly demonstrate that both the activity and stability in ORR can be greatly improved by integrating graphene with $\mathrm{Pt}$ HCCs, as the graphene enhances the conductivity, promotes the catalyst-electrode adhesion, and suppresses the agglomeration and the oxidation erosion of nanocatalysts ${ }^{4,12,30-37,47-59}$.

\section{Discussion}

We have developed a modified protocol to synthesize well-defined nanocrystals with a highly concave cubic profile. The Pt HCC nanocrystals exhibit excellent activity and durability, in a sharp contrast to the commercial $\mathrm{Pt} / \mathrm{C}$ catalysts. Enabled by assembly with $\mathrm{rGO}$, the $\mathrm{Pt}$ HCCs-rGO hybrid structure displays mass activity of $-0.847 \mathrm{~A}$ $\mathrm{mg}_{\mathrm{Pt}}{ }^{-1}$ and specific activity of $-1.02 \mathrm{~mA} \mathrm{~cm}^{-2}$, which are 5.7 times and 7.0 times greater than those of $\mathrm{Pt} / \mathrm{C}$, respectively. It is an impressive record that the half-wave potential reaches as high as $0.967 \mathrm{~V}$, which stands as $63 \mathrm{mV}$ greater than that of $\mathrm{Pt} / \mathrm{C}$. This value is slightly superior to the record obtained in porous Pt-Ni catalysts in $0.1 \mathrm{M}$ $\mathrm{HClO}_{4}$ solution in literature. It clearly demonstrates that the pure $\mathrm{Pt}$ catalysts may achieve high activity and durability without the need of formulating alloys. This work is expected to pave the way to investigate the structure-activity relationship using a pure Pt model. It is anticipated that the findings here will be able to implement in the Ptbased alloy systems for further performance improvement.

\section{Methods}

Synthesis of Pt HCC nanocrystals. In a typical synthesis, $5 \mathrm{~mL}$ ethylene glycol (EG, Aladdin) was hosted in a 25-mL, 3-neck flask (equipped with a reflux condenser and a Teflon-coated magnetic stirring bar) and heated in air under magnetic stirring at $116^{\circ} \mathrm{C}$. Meanwhile, $0.0677 \mathrm{~g}$ of $\mathrm{H}_{2} \mathrm{PtCl}_{6}$ (Aldrich) and $7.5 \mathrm{mmol}$ of $\mathrm{HCl}$ were dissolved in $3 \mathrm{~mL} \mathrm{EG}$; $0.600 \mathrm{~g}$ of $\mathrm{KBr}$ and $0.0916 \mathrm{~g}$ of poly(vinyl pyrrolidone) (PVP, M.W. $\approx 55,000$, Sigma-Aldrich) were dissolved in $3 \mathrm{~mL}$ deionized (DI) water at room temperature. The aqueous stock solution was then quickly injected into the flask, followed by the addition of EG stock solution. The reaction mixture was heated at $116^{\circ} \mathrm{C}$ for $24 \mathrm{~h}$, by continuously bubbling a mixed gas of Ar and air at the ratio of $0.8-1.2$. The product was collected by centrifugation and washed with acetone once, ethanol three times and DI water four times to remove most of the EG and excess PVP

Assembly of Pt HCCs-rGO hybrid structure. GO was synthesized from natural graphite flakes (325 mesh, Alfa-Aesar) using a modified Hummers method ${ }^{60}$. The GO was exfoliated to form a homogeneous aqueous dispersion $\left(0.25 \mathrm{mg} \mathrm{mL}^{-1}\right)$ with probe sonication (Scientz-IID, China) for $1 \mathrm{~h}$. Subsequently, $100 \mathrm{mg}$ of PVP (M.W $\approx 40,000$, Sigma-Aldrich) was mixed with $25 \mathrm{~mL}$ of the GO dispersion, followed by magnetic stirring at ambient temperature for $24 \mathrm{~h}$. The resulting dispersion was added into $20 \mu \mathrm{L}$ of hydrazine hydrate (85\%) and $100 \mu \mathrm{L}$ of ammonia solution, which was stirred at $95^{\circ} \mathrm{C}$ for $1 \mathrm{~h}$. Finally, the stable black dispersion was centrifuged and washed with acetone once, and then redispersed into $25-\mathrm{mL}$ DI water. The assynthesized PVP-functionalized rGO solution was then mixed with $500 \mu \mathrm{L}$ of Pt HCC aqueous dispersion (containing $18 \mathrm{mg} \mathrm{mL}^{-1} \mathrm{Pt}$ ) and was stirred at ambient temperature for another $12 \mathrm{~h}$. The product was collected by centrifugation and washed with acetone once, ethanol three times and DI water ten times to remove residual PVP.

1. Mallouk, T. E. Fuel-cells-miniaturized electrochemistry. Nature 343, 515-516 (1990).

2. Steele, B. C. H. \& Heinzel, A. Materials for fuel-cell technologies. Nature 414, 345-352 (2001)

3. Greeley, J. et al. Alloys of platinum and early transition metals as oxygen reduction electrocatalysts. Nat. Chem. 1, 552-556 (2009).

4. Stamenkovic, V. R. et al. Trends in electrocatalysis on extended and nanoscale Ptbimetallic alloy surfaces. Nat. Mater. 6, 241-247 (2007).

5. Min, M. K., Cho, J. H., Cho, K. W. \& Kim, H. Particle size and alloying effects of Pt based alloy catalysts for fuel cell applications. Electrochim. Acta. 45, 4211-4217 (2000).

6. Van der Vliet, D. et al. Platinum-alloy nanostructured thin film catalysts for the oxygen reduction reaction. Electrochim. Acta. 56, 8695-8699 (2011).

7. Mazumder, V., Chi, M. F., More, K. L. \& Sun, S. H. Core/shell Pd/FePt nanoparticles as an active and durable catalyst for the oxygen reduction reaction. J. Am. Chem. Soc. 132, 7848-7849 (2010).

8. Stamenkovic, V., Schmidt, T. J., Ross, P. N. \& Markovic, N. M. Surface segregation effects in electrocatalysis: kinetics of oxygen reduction reaction on polycrystalline $\mathrm{Pt}_{3} \mathrm{Ni}$ alloy surfaces. J. Electroanal. Chem. 554, 191-199 (2003).

9. Anderson, A. B. et al. Activation energies for oxygen reduction on platinum alloys: Theory and experiment. J. Phys. Chem. B. 109, 1198-1203 (2005).

10. Wang, C. et al. Monodisperse $\mathrm{Pt}_{3} \mathrm{Co}$ nanoparticles as a catalyst for the oxygen reduction reaction: Size-dependent activity. J. Phys. Chem. C. 113, 19365-19368 (2009).

11. Lim, B. et al. Pd-Pt bimetallic nanodendrites with high activity for oxygen reduction. Science 324, 1302-1305 (2009).

12. Stamenkovic, V. R. et al. Improved oxygen reduction activity on $\mathrm{Pt}_{3} \mathrm{Ni}(111)$ via increased surface site availability. Science 315, 493-497 (2007). 
13. Sasaki, K. et al. Recent advances in platinum monolayer electrocatalysts for oxygen reduction reaction: Scale-up synthesis, structure and activity of Pt shells on Pd cores. Electrochim. Acta. 55, 2645-2652 (2010).

14. Gong, K. P., Su, D. \& Adzic, R. R. Platinum-monolayer shell on $\mathrm{AuNi}_{0.5} \mathrm{Fe}$ nanoparticle core electrocatalyst with high activity and stability for the oxygen reduction reaction. J. Am. Chem. Soc. 132, 14364-14366 (2010).

15. Koenigsmann, C. et al. Enhanced electrocatalytic performance of processed, ultrathin, supported Pd-Pt core-shell nanowire catalysts for the oxygen reduction reaction. J. Am. Chem. Soc. 133, 9783-9795 (2011).

16. Xing, Y. C. et al. Enhancing oxygen reduction reaction activity via Pd-Au alloy sublayer mediation of Pt monolayer electrocatalysts. J. Electroanal. Chem. 1, 3238-3242 (2010).

17. Zhou, W. P. et al. Gram-scale-synthesized $\mathrm{Pd}_{2} \mathrm{Co}$-supported Pt monolayer electrocatalysts for oxygen reduction reaction. J. Phys. Chem. C. 114, 8950-8957 (2010).

18. Snyder, J., Fujita, T., Chen, M. W. \& Erlebacher, J. Oxygen reduction in nanoporous metal-ionic liquid composite electrocatalysts. Nat. Mater. 9, 904-907 (2010).

19. Liang, Y. Y. et al. $\mathrm{Co}_{3} \mathrm{O}_{4}$ nanocrystals on graphene as a synergistic catalyst for oxygen reduction reaction. Nat. Mater. 10, 780-786 (2011).

20. Gong, K. P., Du, F., Xia, Z. H., Durstock, M. \& Dai, L. M. Nitrogen-doped carbon nanotube arrays with high electrocatalytic activity for oxygen reduction. Science 323, 760-764 (2009).

21. Lefevre, M., Proietti, E., Jaouen, F. \& Dodelet, J. P. Iron-based catalysts with improved oxygen reduction activity in polymer electrolyte fuel cells. Science 324, 71-74 (2009).

22. Wu, G., More, K. L., Johnston, C. M. \& Zelenay, P. High-performance electrocatalysts for oxygen reduction derived from polyaniline, iron, and cobalt. Science 332, 443-447 (2011).

23. Kuzume, A., Herrero, E. \& Feliu, J. M. Oxygen reduction on stepped platinum surfaces in acidic media. J. Electroanal. Chem. 599, 333-343 (2007).

24. Macia, M. D., Campina, J. M., Herrero, E. \& Feliu, J. M. On the kinetics of oxygen reduction on platinum stepped surfaces in acidic media. J. Electroanal. Chem. 564, 141-150 (2004).

25. Komanicky, V., Menzel, A. \& You, H. Investigation of oxygen reduction reaction kinetics at (111)-(100) nanofaceted platinum surfaces in acidic media. J. Phys. Chem. B. 109, 23550-23557 (2005).

26. Lim, B. \& Xia, Y. N. Metal nanocrystals with highly branched morphologies. Angew. Chem. Int. Ed. 50, 76-85 (2011).

27. Xia, Y. N., Xiong, Y. J., Lim, B. \& Skrabalak, S. E. Shape-controlled synthesis of metal nanocrystals: simple chemistry meets complex physics? Angew. Chem. Int. Ed. 48, 60-103 (2009).

28. Yu, T., Kim, D. Y., Zhang, H. \& Xia, Y. N. Platinum concave nanocubes with highindex facets and their enhanced activity for oxygen reduction reaction. Angew. Chem. Int. Ed. 50, 2773-2777 (2011).

29. Mahmoud, M. A., Tabor, C. E., El-Sayed, M. A., Ding, Y. \& Wang, Z. L. A new catalytically active colloidal platinum nanocatalyst: the multiarmed nanostar single crystal. J. Am. Chem. Soc. 130, 4590-4591 (2008)

30. Meyer, J. C. et al. The structure of suspended graphene sheets. Nature 446, 60-63 (2007)

31. Novoselov, K. S. et al. Two-dimensional gas of massless Dirac fermions in graphene. Nature 438, 197-200 (2005).

32. Zhang, Y. B., Tan, Y. W., Stormer, H. L. \& Kim, P. Experimental observation of the quantum Hall effect and Berry's phase in graphene. Nature 438, 201-204 (2005).

33. Yang, J., Zang, C. L., Sun, L., Zhao, N. \& Cheng, X. N. Synthesis of graphene/Ag nanocomposite with good dispersibility and electroconductibility via solvothermal method. Mater. Chem. Phys. 129, 270-274 (2011).

34. Eda, G., Fanchini, G. \& Chhowalla, M. Large-area ultrathin films of reduced graphene oxide as a transparent and flexible electronic material. Nat. Nanotechnol. 3, 270-274 (2008).

35. Lee, C., Wei, X., Kysar, J. W. \& Hone, J. Measurement of the elastic properties and intrinsic strength of monolayer graphene. Science 321, 385-388 (2008).

36. Guo, S. J. \& Sun, S. H. FePt nanoparticles assembled on graphene as enhanced catalyst for oxygen reduction reaction. J Am Chem Soc. 134, 2492-2495 (2012)

37. Zhou, M., Zhang, A. H., Dai, Z. X., Feng, Y. P. \& Zhang, C. Strain-enhanced stabilization and catalytic activity of metal nanoclusters on graphene. J. Phys Chem. C 114, 16541-16546 (2010)

38. Ma, L. et al. Control over the branched structures of platinum nanocrystals for electrocatalytic applications. ACS Nano. 6, 9797-9806 (2012).

39. Lindstrom, R. W. et al. Active area determination of porous Pt electrodes used in polymer electrolyte fuel cells: temperature and humidity effects. J. Electrochem. Soc. 157, B1795-B1801 (2010).

40. Lee, S. J. et al. Effects of nafion impregnation on performances of PEMFC electrodes. Electrochim. Acta 43, 3693-3701 (1998).

41. Markus, Nesselberger. et al. The particle size effect on the oxygen reduction reaction activity of Pt catalysts: Influence of electrolyte and relation to single crystal models. J. Am. Chem. Soc. 133, 17428-17433 (2011).
42. Peng, Z. M. \& Yang, H. Synthesis and oxygen reduction electrocatalytic property of Pt-on-Pd bimetallic heteronanostructures. J. Am. Chem. Soc. 131, 7542-7543 (2009).

43. Peng, Z. M. \& Yang, H. Designer platinum nanoparticles: Control of shape, composition in alloy, nanostructure and electrocatalytic property. Nano Today 4 , 143-164 (2009)

44. Clouser, S. J., Huang, J. C. \& Yeager, E. Temperature dependence of the Tafel slope for oxygen reduction on platinum in concentrated phosphoric acid. J. Appl. Electrochem. 23, 597-605 (1993).

45. Ghoneim, M. M., Clouser, S. \& Yeager, E. Oxygen reduction kinetics in deuterated phosphoric acid. J. Electrochem. Soc. 132, 1160-1162 (1985).

46. Hsueh, K. L., Gonzalez, E. R. \& Srinivasan, S. Electrolyte effects on oxygen reduction kinetics at platinum: a rotating-ring disk electrode analysis. Electrochim. Acta 28, 691-697 (1983).

47. Kamat, P. V. Graphene-based nanoarchitectures. Anchoring semiconductor and metal nanoparticles on a two-dimensional carbon support. J. Phys. Chem. Lett. 1, 520-527 (2010)

48. Park, S. \& Ruoff, R. S. Chemical methods for the production of graphenes. Nat Nanotechnol. 4, 217-224 (2009).

49. Li, D., Muller, M. B., Gilje, S., Kaner, R. B. \& Wallace, G. G. Processable aqueous dispersions of graphene nanosheets. Nat. Nanotechnol. 3, 101-105 (2008).

50. Raman, R. K. S. et al. Protecting copper from electrochemical degradation by graphene coating. Carbon 50, 4040-4045 (2012)

51. Tuinstra, F. \& Koenig, J. L. Raman spectrum of graphite. J. Chem. Phys. 53, 1126-1130 (1970).

52. Tung, V. C., Allen, M. J., Yang, Y. \& Kaner, R. B. High-throughput solution processing of large-scale graphene. Nat. Nanotechnol. 4, 25-29 (2009).

53. Stankovich, S. et al. Synthesis of graphene-based nanosheets via chemica reduction of exfoliated graphite oxide. Carbon 45, 1558-1565 (2007).

54. Zhu, C. Z., Guo, S. J., Fang, Y. X. \& Dong, S. J. Reducing sugar: new functional molecules for the green synthesis of graphene nanosheets. ACS Nano 4 2429-2437 (2010).

55. Markovic, N. M., Gasteiger, H. A., Grgur, B. N. \& Ross, P. N. Oxygen reduction reaction on Pt (111): effects of bromide. J. Electroanal. Chem. 467, 157-163 (1999).

56. Zhang, J., Sasaki, K., Sutter, E. \& Adzic, R. R. Stabilization of platinum oxygenreduction electrocatalysts using gold clusters. Science 315, 220-222 (2007)

57. Li, Y. J. et al. Stabilization of high-performance oxygen reduction reaction $\mathrm{Pt}$ electrocatalyst supported on reduced graphene oxide/carbon black composite. J. Am. Chem. Soc. 134, 12326-12329 (2012).

58. Kim, K. S. et al. Large-scale pattern growth of graphene films for stretchable transparent electrodes. Nature 457, 706-710 (2009).

59. Geim, A. K. Graphene: Status and prospects. Science 324, 1530-1534 (2009).

60. Hummers, W. S. \& Offeman, R. E. Preparation of graphitic oxide. J. Am. Chem. Soc. 80, 1339-1339 (1958).

\section{Acknowledgements}

This work was financially supported by the National Natural Science Foundation of China (NSFC) (No. 21101145), the Recruitment Program of Global Experts, the CAS Hundred Talent Program, and the Fundamental Research Funds for the Central Universities (Nos. WK2060190025, WK2340000017).

\section{Author contributions}

Y.X. conceived the research and designed the experiments. Y.X., C.W. and L.L. analyzed the data. C.W. performed most of the experiments. L.M. and S.B. synthesized the materials. M.Z. and R.L. performed the TEM studies. Y.X. and C.W. co-wrote the paper. All authors discussed the results and commented on the manuscript.

\section{Additional information}

Supplementary information accompanies this paper at http://www.nature.com/ scientificreports

Competing financial interests: The authors declare no competing financial interests.

How to cite this article: Wang, C.M. et al. A unique platinum-graphene hybrid structure for high activity and durability in oxygen reduction reaction. Sci. Rep. 3, 2580; DOI:10.1038/ srep02580 (2013)

This work is licensed under a Creative Commons AttributionNonCommercial-NoDerivs 3.0 Unported license. To view a copy of this license, visit http://creativecommons.org/licenses/by-nc-nd/3.0 